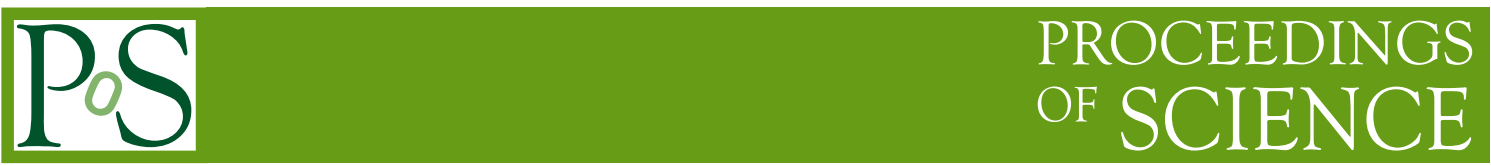

\title{
INTEGRAL Studies of Nucleosynthesis Lines
}

\author{
M. Leising ${ }^{*+}$ \\ Clemson University \\ E-mail: lmark@clemson.edu \\ R. Diehl \\ Max Planck Institut für extraterrestrische Physik, Garching, Germany \\ E-mail: rod@mpe.mpg.de
}

The SPI Ge spectrometer on INTEGRAL continues to collect data on astrophysically-important $\gamma$-ray lines from decays of ${ }^{26} \mathrm{Al}$ and ${ }^{60} \mathrm{Fe}$, and positron annihilation. ${ }^{44} \mathrm{Ti}$ decay lines from Cas A have been observed with the IBIS imager, but the lack of other ${ }^{44} \mathrm{Ti}$ remnants is a mystery. The ${ }^{26} \mathrm{Al} \gamma$-ray line is now measured throughout the Galaxy, tracing the kinematics of interstellar gas near massive stars, and highlighting regions of interest, such as groups of massive stars in Cygnus and even more nearby regions, such as in Orion. The detection of ${ }^{60} \mathrm{Fe}$ radioactivity lines at the level of $15 \%$ of the ${ }^{26} \mathrm{Al}$ flux presents a challenge both for measurement and explanation. Positron annihilation emission from the nucleosynthesis regions within the Galactic plane appears to be mainly from ${ }^{26} \mathrm{Al}$ and other supernova radioactivity, while the bulge region's positron annihilation brightness remains a puzzle. The continuing accumulation of large exposures to the central Galaxy, and the anticipated continuing availability of INTEGRAL for rare nearby explosive events promise further improvements in our understanding of all these nucleosynthesis sources.

7th INTEGRAL Workshop

September 8-11 2008

Copenhagen, Denmark

\footnotetext{
* Speaker.

${ }^{\dagger}$ Supported by NASA

${ }^{\star}$ from studies with SPI on INTEGRAL, supported by ESA and its member countries
} 


\section{Introduction}

Setting the stage for INTEGRAL, the study of celestial nuclear $\gamma$-ray lines (apart from the nuclear contribution to the galactic positrons) began with the $\gamma$-ray spectrometer aboard the third High-Energy Astronomy Observatory (HEAO C-1). It detected the $1.809 \mathrm{MeV}$ line of ${ }^{26} \mathrm{Al}$ decay in its two two-week scans along the galactic plane in 1979 and 1980 [1, 2]. The Solar Maximum Mission Gamma-Ray Spectrometer's exceptional stability and nearly a decade of tracking the Sun around sky led to improved measurements of the ${ }^{26} \mathrm{Al}$ line $[3,4]$. Both the HEAO C-1 and SMM/GRS data set interesting upper limits with non-detections of line emission from ${ }^{44} \mathrm{Ti}$ and ${ }^{60} \mathrm{Fe}[1,5]$. The fortuitous SN 1987A provided an opportunity to study a core-collapse supernova up close, and even though it could not be pointed at the supernova, the SMM/GRS was able to detect several lines of ${ }^{56} \mathrm{Co}$ decay $[6,7]$. The lines emerged earlier than expected, indicating mixing of a few percent of the core radioactivity into the hydrogen envelope or beyond.

The next advances were provided by the Compton Gamma Ray Observatory. To study $\gamma$ ray lines, it carried the Oriented Scintillation Spectrometer Experiment (CGRO/OSSE) and the Compton Telescope (CGRO/COMPTEL.) The CGRO/OSSE produced the first maps of the diffuse electron-positron annihilation radiation and measured ${ }^{57} \mathrm{Co}$ decay in SN 1987A [8]. The CGRO/COMPTEL made the first map of the $1.809 \mathrm{MeV}{ }^{26} \mathrm{Al}$ decay photons (Fig. 1) as well as maps of diffuse continuum emission.

The currently operating INTEGRAL instruments SPI and IBIS both use code masks for imaging and background estimation. While not optimal for measuring diffuse emission, both provide unique capabilities. SPI provides an unprecedented combination of energy resolution and sensitivity; IBIS angular resolution and sensitivity at hard X-ray energies. Both of these instruments are making significant contributions to $\gamma$-ray line studies, which we review below.

\section{Diffuse Emission}

\section{$2.1{ }^{26} \mathrm{Al}$}

The $\gamma$-ray line from ${ }^{26} \mathrm{Al}$ radioactivity $\left(\tau=1.04 \mathrm{My}, \mathrm{E}_{\gamma}=1808.65 \mathrm{keV}\right)$ may be considered the most direct proof of ongoing nucleosynthesis in the current epoch of the Galaxy's evolution. The history of ${ }^{26} \mathrm{Al}$ line observations up to the epoch of the Compton Observatory has been described earlier [9]. ${ }^{26} \mathrm{Al}$ production as imaged by COMPTEL (see Fig. 1 [left]) occurs throughout the Galaxy, with apparent concentrations in regions hosting young and massive stars such as in Cygnus. The patchy distribution of ${ }^{26} \mathrm{Al}$ emission along the plane of the Galaxy could partly be instrumental, but argues for massive stars as dominant sources of Galactic ${ }^{26} \mathrm{Al}$. This is consistent with estimates from the ionizing radiation by those same stars [10]. Adopting plausible large-scale source distributions, the total amount of ${ }^{26} \mathrm{Al}$ in the Galaxy was estimated as $2-3 \mathrm{M}_{\odot}$. Massive stars, through core-collapse supernovae and Wolf-Rayet phases, eject ${ }^{26} \mathrm{Al}$ into the interstellar medium, where it decays to produce the observed emission. Typical ejection velocities are $1000 \mathrm{~km} \mathrm{~s}^{-1}$ or more. Ejecta are slowed by interaction with circumstellar and interstellar matter. Depending on when the ${ }^{26} \mathrm{Al}$ is slowed, one would expect more or less Doppler broadening of the observed ${ }^{26} \mathrm{Al}$ line. Therefore, the ${ }^{26} \mathrm{Al}$ line provides a diagnostic of the mean environment around massive stars, which 

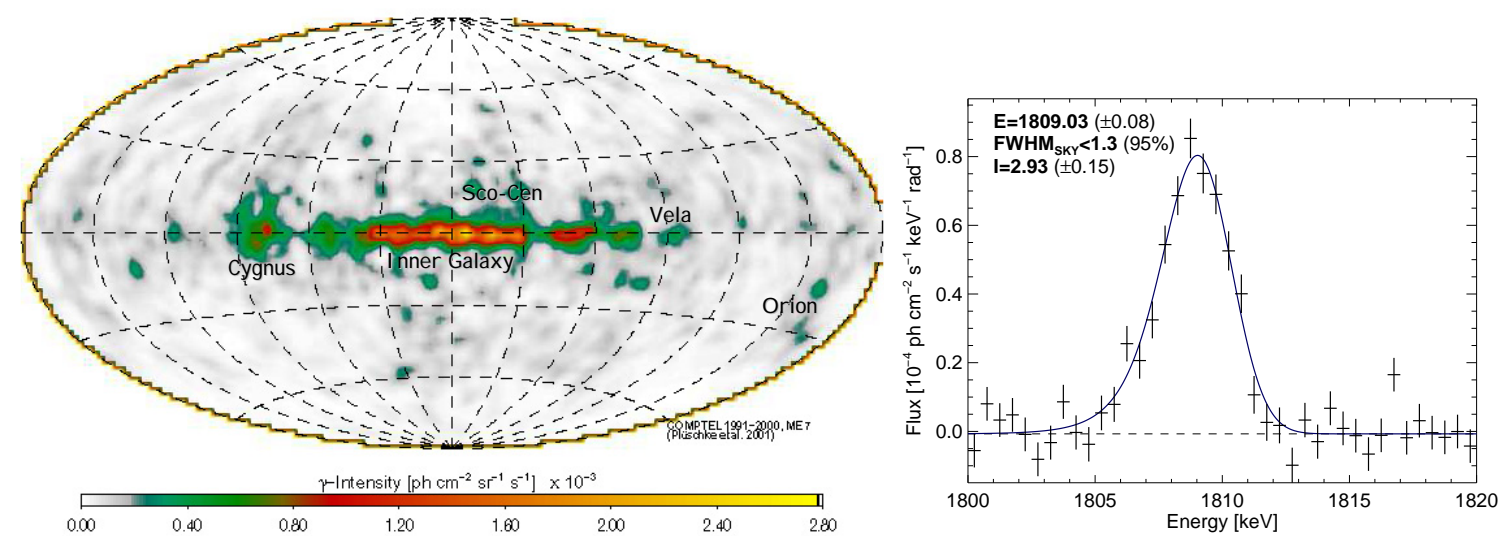

Figure 1: ${ }^{26} \mathrm{Al}$ radioactivity from the Galaxy, as seen by the Compton Observatory and INTEGRAL. (Left:): The ${ }^{26} \mathrm{Al}$ image from 9 years of sky survey with COMPTEL shows somewhat patchy emission along the plane of the Galaxy [13].(Right:): The ${ }^{26} \mathrm{Al}$ spectrum as measured by INTEGRAL/SPI shows the line to be rather narrow $[14,15]$

is rather unique: Such interstellar gas is dilute and ionized, so radiative effects are sparse; nevertheless, this "hot phase" of the ISM has important implications for galactic structure and evolution. The GRIS balloone-borne Ge spectrometer instrument had reported such Doppler broadening [11], but the associated mean ${ }^{26} \mathrm{Al}$ nucleus velocities at the time of decay of about $500 \mathrm{~km} \mathrm{~s}^{-1}$ appeared to be implausibly large [12]. One of the main goals for INTEGRAL's high-resolution Ge spectrometer instrument SPI therefore was a clear spectroscopic measurement of the ${ }^{26} \mathrm{Al}$ line shape to assess the kinematics of ${ }^{26} \mathrm{Al}$ throughout the Galaxy.

The ${ }^{26} \mathrm{Al}$ line was found to be rather narrow with first observations of INTEGRAL [14], confirming the measurement made with the Ge spectrometer on the RHESSI solar satellite [16]. Although the line was not clearly resolved, it appeared somewhat broadener than instrumental resolution, but far less than the $5.4 \mathrm{keV}$ broadening of the earlier result [11]. Subsequent INTEGRAL measurements added precision and spatial information on the ${ }^{26} \mathrm{Al}$ line parameters (Fig. 2). Observations of the inner Galaxy showed shifts in the line centroid, which plausibly followed the Doppler shifts expected from the large-scale galactic differential rotation [17]. With more data, line centroid determinations can be made for different viewing directions (Fig. 2). Recent results suggest an asymmetry, with significantly-blueshifted ${ }^{26} \mathrm{Al}$ line emission towards the fourth quadrant of the Galaxy (i.e., negative longitudes). The value of the blue-shift appears on the high side of what is expected from differential rotation, and may reflect peculiar bulk motion such as expected within the bar of the Galaxy. On the other hand, there are apparently no corresponding redshifts of the ${ }^{26} \mathrm{Al}$ line at correspondingly-positive longitude values, i.e. in the Galaxy's first quadrant. In terms of ${ }^{26} \mathrm{Al}$ emission, the inner Galaxy appears asymmetric; peculiar bulk motion could compensate for differential large-scale rotation here, or the differences in inner spiral-arm structures may be responsible for these results.

As the ${ }^{26} \mathrm{Al}$ signal weakens away from the inner Galaxy, it becomes more difficult to determine separately the line shift and width per longitude bin. Analyzing 30-degree segments, it seems that towards the Aquila direction $\left(l \simeq 25^{\circ}\right)$ the measured ${ }^{26} \mathrm{Al}$ line is broader than seen toward other directions. If one such region of a particularly young age (below about 5 Myrs) dominates the 

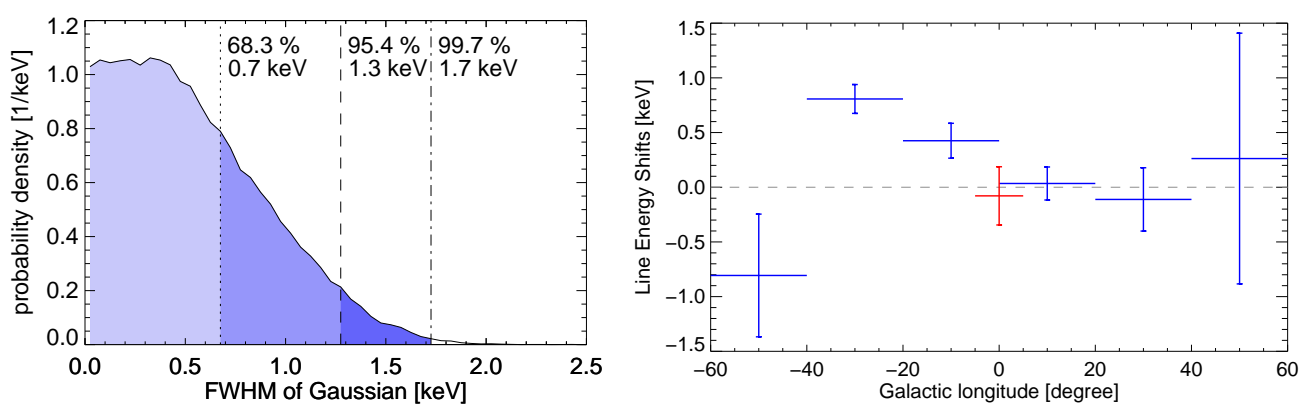

Figure 2: ${ }^{26} \mathrm{Al}$ line details from INTEGRAL: (Left:) The probability distribution for additional broadening of the ${ }^{26} \mathrm{Al}$ line, beyond the instrumental width [15]. (Right:) The ${ }^{26} \mathrm{Al}$ line centroid positions for different directions along the plane of the Galaxy show an expected blue-shift from large-scale galactic rotation in the fourth quadrant; a corresponding redshift in the first quadrant is not clear ([15]; see also [17]).

emission, a larger line width would be plausible, from increased turbulence of the ISM around groups of massive stars. The integrated ${ }^{26} \mathrm{Al}$ signal observed with INTEGRAL now has reached a significance $(28 \sigma ;[15])$ similar to what COMPTEL obtained in its 9-year mission, making possible improved tests of kinematics of ${ }^{26} \mathrm{Al}$ nuclei at the time of their decay. The instrumental line width of $3 \mathrm{keV}$ FWHM limits such tests; moreover the periodic annealings and intervening cosmic-ray induced $10 \%$ degradations of detector resolution result in a time-variable detector resolution over the years of accumulated observations. Since these instrumental effects are well-calibrated, we can compare the observed line shape to the expected one from instrumental properties, and parametrize the difference in terms of an additional (assumed-Gaussian) astrophysical broadening. Fig 2 (left) shows the probability distribution as obtained from this test. Clearly, there is no positive broadening measured; the probability distribution peaks at small values near zero. Yet the shape, with an indicated plateau up to a few tenths of a $\mathrm{keV}$, hints towards a small broadening of $0.3-0.4 \mathrm{keV}$, which would correspond to a velocity of $50 \mathrm{~km} \mathrm{~s}^{-1}$. The probability distribution provides a strict upper limit of $1.3 \mathrm{keV}$ (at $95 \%$ probability) for additional broadening, which limits ${ }^{26} \mathrm{Al}$ velocities to less than $150 \mathrm{~km} \mathrm{~s}^{-1}$ on average. Note that this conversion to velocity limits assumes an isotropic and Gaussian distribution of velocities, such as expected from turbulence in the interstellar medium. Also, the underlying spectrum is obtained from integration over the entire inner Galaxy, which may include regions of different intrinsic turbulence plus the bulk motion from the Galaxy's differential large-scale rotation. In summary, large kinematic broadening in the $100 \mathrm{~km} \mathrm{~s}^{-1}$ range such as discussed ten years ago are excluded, while INTEGRAL's capability to measure velocities down almost to average stellar velocities provides an interesting and unexpected perspective.

Clearly, the integration over many possibly unrelated source regions limits the impact of ${ }^{26} \mathrm{Al}$ results obtained so far. Only in the Cygnus region may one safely assume a coherent and localized single source region along the line of sight, which also has a strong ${ }^{26} \mathrm{Al}$ signal, on the order of $15 \sigma$ [18]. However, the INTEGRAL exposure of the inner Galaxy now is approximately similar to the COMPTEL all-sky survey (1991-2000). With similar spatial resolutions of INTEGRAL/SPI and COMPTEL $\left(2.7^{\circ}\right.$ versus $3.6^{\circ} \mathrm{FWHM}$, respectively), this allows study of specific regions of interest for local line shape diagnostics, with years of INTEGRAL observations still to be analyzed, and still to be acquired. The peculiar line width towards the Aquila region, and isolation of the ${ }^{26} \mathrm{Al}$ 
signal from the nearby Sco-Cen association (distance 100-150 pc) are on the horizon. It will be important that such observations are taken under well-controlled background and spectral-response conditions, so that instrumental limitations are minimized. The Orion region presents the most nearby concentration of young massive stars at about $450 \mathrm{pc}$ distance, well isolated on the sky with respect to other Galactic ${ }^{26} \mathrm{Al}$ sources, and marginally detected with COMPTEL (Fig. 1) [19]. Similar to Cygnus, or even better, is our knowledge about the stellar census of this region. But, being located towards the outer Galaxy with correspondingly lower space density of candidate hard X-ray sources, INTEGRAL's observing program devoted just as much exposure to Orion so that confirmation or not of the weak COMPTEL ${ }^{26} \mathrm{Al}$ signal may be expected. These data are being analyzed, and a positive ${ }^{26} \mathrm{Al}$ detection would likely prompt more observations, for a determination of bulk ${ }^{26} \mathrm{Al}$ velocity of Orion. This is of interest because there is a large interstellar cavity in the foreground of the Orion ${ }^{26} \mathrm{Al}$ sources, which plausibly could cause deviations from sphericallysymmetric ${ }^{26} \mathrm{Al}$ dispersion around its sources.

\section{$2.2{ }^{60} \mathrm{Fe}$}

Stellar nucleosynthesis models have shown that the long-lived $\gamma$-ray emitting radioactive isotope ${ }^{60} \mathrm{Fe}$ is synthesized within the convective envelopes of massive-stars. For stars exceding $8-10$ $\mathrm{M}_{\odot}$, these ${ }^{60} \mathrm{Fe}$-rich ashes would be ejected in the terminal core-collapse supernova explosion. The details are complex, and how shell burning regions develop and extinguish, and which neutronproducing reactions are important are uncertain. Yields of radioactive ${ }^{26} \mathrm{Al}$ and ${ }^{60} \mathrm{Fe}$ are very sensitive to such detail, however [20]. Because of the dominant massive-star origin of ${ }^{26} \mathrm{Al}$, the $\gamma$-ray flux ratio from the two isotopes constitutes an interesting global test of massive-star nucleosynthesis models.

Following many reported non-detections and upper limits, a hint of ${ }^{60} \mathrm{Fe} \gamma$-rays was found with RHESSI [21]. That signal corresponded to $16 \pm 5 \%$ of the ${ }^{26} \mathrm{Al}$ flux, consistent with earlier theoretical predictions of $15 \%$ [22]. Later studies of massive-star nucleosynthesis tended to predict larger ratios of ${ }^{60} \mathrm{Fe}$ versus ${ }^{26} \mathrm{Al}$, as progenitor evolution and wind models[23], as well as nuclearreaction rates [24], were updated. Nucleosynthesis calculations [20, 24] generally still fall on the higher side of the original prediction, but are consistent, given the substantial uncertainties in such models. Uncertainties arise mainly from stellar structure, as establishment of suitable convectiveburning regions is sensitive to stellar rotation, which in turn is affected by the mass loss history during evolution. Uncertainties on nuclear cross sections involve ${ }^{26} \mathrm{Al}$ destruction though $\mathrm{n}$ capture, and n-capture on unstable ${ }^{59} \mathrm{Fe}$ and on ${ }^{60} \mathrm{Fe}$ itself.

Confirmation of the RHESSI ${ }^{60} \mathrm{Fe}$ signal was reported from first-year INTEGRAL/SPI data [25], although features from a nearby instrumental line indicated systematics issues. In a recent analysis of more data, a significant ${ }^{60} \mathrm{Fe}$ signal (at $5 \sigma$ ) was found, with somewhat reduced systematic effects from instrumental background. This underlying background is being investigated, specific signatures within the 19-detector Ge camera of SPI are being exploited to discriminate internal versus celestial $\gamma$-rays on their respective modulation time scales. The INTEGRAL/SPI reported ${ }^{60} \mathrm{Fe} /{ }^{26} \mathrm{Al} \gamma$-ray flux ratio is now $0.14 \pm 0.06$. Formally, there is agreement between observations and models (see Fig. 3), but more can be learned as uncertainties in each area are revisited and re-assessed. We also hope to exploit INTEGRAL's spatial resolution, towards determination of spatially-resolved ${ }^{60} \mathrm{Fe}$ to ${ }^{26} \mathrm{Al}$ ratios. It seems feasible to obtain values for the different Galactic 

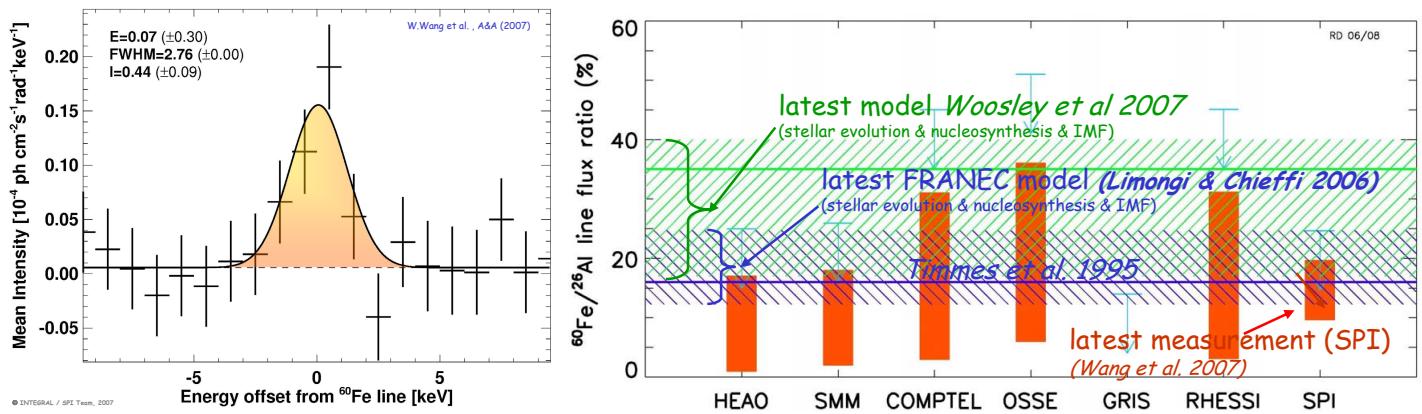

Figure 3: (Left:) The ${ }^{60} \mathrm{Fe}$ measurement with INTEGRAL . Superpositions of both lines of ${ }^{60} \mathrm{Fe}$ at their nominal energies of 1173 and $1332 \mathrm{keV}$, respectively, show a weak signal from ${ }^{60} \mathrm{Fe}$ decay. (Right:) The set of ${ }^{60} \mathrm{Fe} /{ }^{26} \mathrm{Al}$ ratio measurements by different experiments and the ranges of current theoretical predictions appear consistent. Deeper investigations of uncertainties in measurement and model may show if observations are significantly lower than predictions.

quadrants, and the ${ }^{60} \mathrm{Fe}$ limit for the ${ }^{26} \mathrm{Al}$-bright Cygnus region will provide another interesting constraint because, rather than being in steady state, here ${ }^{26} \mathrm{Al}$ from rather young massive-star groups is observed.

\section{Specific Sources}

\section{1 ${ }^{44}$ Ti Supernova Remnants}

Given a galactic supernova rate of $2-3$ per century and a ${ }^{44} \mathrm{Ti}\left({ }^{44} \mathrm{Ti} \frac{\tau=87 \mathrm{y}}{68,78 \mathrm{keV}}{ }^{44} \mathrm{Sc} \frac{\tau=5.7 \mathrm{~h}}{1156 \mathrm{keV}}{ }^{44} \mathrm{Ca}\right)$ yield of nearly $10^{-4} \mathrm{M}_{\odot}$ per event, it is reasonable to expect to find a few remnants in the inner Galaxy detectable at line flux levels near $10^{-4} \mathrm{~cm}^{-2} \mathrm{~s}^{-1}$. Increasingly sensitive searches found none there [26, 5], but the Cas A supernova remnant was detected in the $1.157 \mathrm{MeV}$ line [27]. This has been confirmed by detections of the lower energy lines [28, 29], and now all flux measurements are consistent with $\simeq 2.510^{-5} \mathrm{~cm}^{-2} \mathrm{~s}^{-1}$. These verify the idea that ${ }^{44} \mathrm{Ti}$ is synthesized in the alpharich freezeout of nuclear statistical equilibrium, which occurs in material ejected by core-collapse supernovae. Such observations might shed light on the role of asymmetries in the explosion and on the mechanism of ejection. INTEGRAL/SPI attempts to do spectroscopy on the Cas A ${ }^{44} \mathrm{Ti}$ lines, with a chance to make use of simultaneous measurements of all three decay lines with the same instrument, so far yields only upper limits to the line fluxes. That for the $1.157 \mathrm{MeV}$ line implies a lower limit to the expansion velocity of $\sim 500 \mathrm{~km} \mathrm{~s}^{-1}$ [30].

The lack of other detectable remnants is quite puzzling viewed in any of several ways. Star formation is concentrated in the inner Galaxy, and current $\gamma$-ray line surveys [31] can detect typical remnants at the distance of the galactic center for a few half-lives of ${ }^{44} \mathrm{Ti}$, so the probability that the brightest remnant is in the outer Galaxy, at a distance of $3.4 \mathrm{kpc}$ and of an age 320 years is very improbable, assuming Cas A's ${ }^{44} \mathrm{Ti}$ yield is not much different than the average. Apart from Cas A, current estimates of supernova rates and yields suggest that at least a few remnants should be detected in the inner Galaxy at IBIS hard X-ray sensitivity [32], but even those rates and yields fall short of the expected ${ }^{44} \mathrm{Ca}$ production rates required to explain the solar ${ }^{44} \mathrm{Ca}$ abundance [5, 22]. A 
scenario that reproduces the solar abundance of ${ }^{44} \mathrm{Ca}$, if made as ${ }^{44} \mathrm{Ti}$, yields even more detectable remnants.

The solution of this puzzle is not clear. Perhaps supernova ${ }^{44} \mathrm{Ti}$ yields vary greatly, and the outliers on the high side contribute most of the ${ }^{44} \mathrm{Ti}$. However, Cas A, whose yield, $1.410^{-4}$ $\mathrm{M}_{\odot}$ is higher than theoretical calculations, is not extreme enough. We would still require 1-2 such supernovae per century, a few of which should be detectable. A plausible solution is that much higher yield ${ }^{44} \mathrm{Ti}$ sources, which are very rare, e.g., He-triggered low-mass thermonuclear supernovae [33], produce the time-averaged ${ }^{44} \mathrm{Ca}$ rate, but none has occurred in the Galaxay in recent centuries. No such source has been recognized among extragalactic supernovae or galactic remnants. Such objects should be visible in the K X-rays of radioactive ${ }^{59} \mathrm{Ni}(\tau=75 \mathrm{ky})$ decay [34] if they exist.

There is also evidence that ${ }^{44} \mathrm{Ti}$ is present in the ejecta of SN 1987A [35] at a mass somewhat less than that in Cas A. The implied $\gamma$-ray line flux could be $310^{-6} \mathrm{ph}^{-2} \mathrm{sm}^{-1}$, which is undetectable to current instruments, but could be measured by near-term instruments with hard X-ray optics or much improved Compton telescopes.

\subsection{Supernovae Ia and Novae}

From general considerations, it was thought that the objects best studied by $\gamma$-ray lines would be the profoundly thermonuclear objects, classical novae and Type Ia supernovae, rather than the core-collapse supernovae for which the nuclear reactions are a side effect. However, we have definitively detected neither a nova nor a thermonuclear supernova. The reason is simply luck; none have occurred within the suitable volumes sampled by our instruments.

Type Ia supernovae are good standard candles because they are efficient thermonuclear bombs, producing typically $0.6 \mathrm{M}_{\odot}$ of ${ }^{56} \mathrm{Ni}$ whose decay powers the visible display. Their kinetic energies are high enough that the $\gamma$-ray escape is significant after one month. The best limit on ${ }^{56} \mathrm{Co}$ lines from a SN Ia was set by the SMM/GRS for the nearby SN 1986G, which found to have ejected less than $0.4 \mathrm{M}_{\odot}$ of ${ }^{56} \mathrm{Ni}[36]$. This was a somewhat under-luminous Type Ia, for which that ${ }^{56} \mathrm{Ni}$ mass is not now thought to be extreme. The high-luminosity SN Ia prototype, SN 1991T, was the first prospect for CGRO, but its distance of 13-14 Mpc meant that only upper limits were achieved [37, 38], however one analysis method showed possible ${ }^{56} \mathrm{Co}$ line features [39]. SN 1998bu was only slightly closer, and also yielded only upper limits [40]. No SN Ia has been closer in the INTEGRAL era, though SN 2003gs was observed early in the mission. No lines were seen. With recently obtained sensitivity to broad lines, SPI can detect typical SN Ia models in ${ }^{56} \mathrm{Co}$ lines to distance of 6-7 Mpc, which occurs about once per twenty-five years. IBIS can possibly detect the $80-150 \mathrm{keV}$ Compton continuum to distance of 9-10 Mpc, expected about once per decade.

Classical novae, envelope thermonuclear flashes on accreting white dwarfs, were long suspected to be bright $\gamma$-ray line sources [41, 42] Proton rich nuclei, including $\gamma$-ray emitters ${ }^{22} \mathrm{Na}$ and ${ }^{26} \mathrm{Al}$ and short-lived positron emitters ${ }^{13} \mathrm{~N}$ and ${ }^{18} \mathrm{~F}$, should be ejected, but yields are uncertain mainly because the underlying models are uncertain. Searches for ${ }^{22} \mathrm{Na}$ lines have so far proved fruitless, both blind and of nearby novae [1, 43, 44]. The nova contribution to the diffuse ${ }^{26} \mathrm{Al}$ emission is unknown, but is probably not dominant given the lack of ${ }^{26} \mathrm{Al}$ in the bulge. The electron-positron annihilation emission over the first hours after outburst would be an excellent diagnostic of the burning and transport of envelope material. The models have become somewhat 
more pessimistic in recent years [45]. As the onset of the runaway is unpredictable, a wide field monitor with good background stability is essential. The FERMI $\gamma$-ray burst monitor (GBM) might serve this purpose in the immediate future.

\section{Summary}

Gamma-ray lines from radioactive decay of cosmic nuclei have provided astrophysics with direct proof of ongoing nucleosynthesis in the present-day universe. Although the number of different isotopes available for such study is limited, these provide key calibration points for models of nucleosynthesis in stars and supernovae. ${ }^{44} \mathrm{Ti}$ is beginning to yield unique insights into the interior processes of massive-star gravitational collapses, with potential further insight into asymmetrical features such as jets, while corresponding measurements with Ni isotopes on thermonuclear supernova processes have not had the luck of a sufficiently nearby event. Diffuse $\gamma$-ray emission in the Galaxy from decay of radioactive ${ }^{26} \mathrm{Al}$ and ${ }^{60} \mathrm{Fe}$ all provide tests of our models of massive-star interior structure and nucleosynthesis. INTEGRAL measurements, in most cases with just a few years of data, have advanced our understanding of each of these topics. It continues to provide a unique capability for study of $\gamma$-ray line emission, which will not be replaced in the foreseeable future.

\section{References}

[1] W. A. Mahoney, J. C. Ling, A. S. Jacobson and R. E. Lingenfelter, ApJ 262 (Nov., 1982) 742-+.

[2] W. A. Mahoney, J. C. Ling, W. A. Wheaton and A. S. Jacobson, ApJ 286 (Nov., 1984) 578-585.

[3] G. H. Share, R. L. Kinzer, J. D. Kurfess et. al., ApJ 292 (May, 1985) L61-L65.

[4] M. J. Harris, G. H. Share, M. D. Leising et. al., ApJ 362 (Oct., 1990) 135-146.

[5] M. D. Leising and G. H. Share, ApJ 424 (Mar., 1994) 200-207.

[6] S. M. Matz, G. H. Share, M. D. Leising et. al., Nature 331 (1988) 416-418.

[7] M. D. Leising and G. H. Share, ApJ 357 (July, 1990) 638-648.

[8] J. D. Kurfess, W. N. Johnson, R. L. Kinzer et. al., ApJ 399 (Nov., 1992) L137-L140.

[9] N. Prantzos and R. Diehl, Phys. Rep. 267 (Mar., 1996) 1-69.

[10] J. Knödlseder ApJ 510 (Jan., 1999) 915-929.

[11] J. E. Naya, S. D. Barthelmy, L. M. Bartlett et. al., Nature 384 (Nov., 1996) 44-46.

[12] W. Chen, R. Diehl, N. Gehrels et. al., in The Transparent Universe (C. Winkler, T. J.-L. Courvoisier and P. Durouchoux,, eds.) vol. 382, pp. 105-+, 1997.

[13] S. Plüschke, R. Diehl, V. Schönfelder et. al., in Exploring the Gamma-Ray Universe (A. Gimenez, V. Reglero and C. Winkler,, eds.) vol. 459, pp. 55-58, Sept., 2001.

[14] R. Diehl, J. Knödlseder, G. G. Lichti et. al., A\&A 411 (Nov., 2003) L451-L455 [arXiv:astro-ph/0309097].

[15] W. Wang, M. G. Lang, R. Diehl et. al., A\&A 496 (Mar., 2009) 713-724 [0 902 . 0211].

[16] D. M. Smith ApJ 589 (May, 2003) L55-L58 [arXiv : astro-ph/ 0304508 ]. 
[17] R. Diehl, H. Halloin, K. Kretschmer et. al., Nature 439 (Jan., 2006) 45-47 [arXiv:astro-ph/0601015].

[18] J. Knödlseder, M. Valsesia, M. Allain et. al., in 5th INTEGRAL Workshop on the INTEGRAL Universe (V. Schoenfelder, G. Lichti and C. Winkler,, eds.) vol. 552, pp. 33-+, Oct., 2004.

[19] R. Diehl New Astronomy Review 46 (July, 2002) 547-552.

[20] A. Chieffi and M. Limongi, New Astronomy Review 46 (July, 2002) 459-462.

[21] D. M. Smith in 5th INTEGRAL Workshop on the INTEGRAL Universe (V. Schoenfelder, G. Lichti and C. Winkler,, eds.) vol. 552, pp. 45-+, Oct., 2004.

[22] F. X. Timmes, S. E. Woosley, D. H. Hartmann and R. D. Hoffman, ApJ 464 (1996) 332-+.

[23] N. Prantzos A\&A 420 (June, 2004) 1033-1037 [arXiv:astro-ph/ 0402198 ].

[24] S. E. Woosley and A. Heger, Phys. Rep. 442 (Apr., 2007) 269-283 [arXiv: astro-ph/0 02176 ].

[25] M. J. Harris, J. Knödlseder, P. Jean et. al., A\&A 433 (Apr., 2005) L49-L52 [arXiv:astro-ph/0502219].

[26] W. A. Mahoney, J. C. Ling, W. A. Wheaton and J. C. Higdon, ApJ 387 (1992) 314-319.

[27] A. F. Iyudin, R. Diehl, H. Bloemen et. al., A\&A 284 (1994) L1-L4.

[28] J. Vink, J. M. Laming, J. S. Kaastra et. al., ApJ 560 (Oct., 2001) L79-L82 [arXiv:astro-ph/0107468].

[29] M. Renaud, J. Vink, A. Decourchelle et. al., ApJ 647 (Aug., 2006) L41-L44 [arXiv:astro-ph/0606736].

[30] P. Martin, J. Knödlseder, J. Vink et. al., A\&A in press (2009).

[31] M. Renaud, F. Lebrun, J. Ballet et. al., in 5th INTEGRAL Workshop on the INTEGRAL Universe (V. Schoenfelder, G. Lichti and C. Winkler,, eds.) vol. 552, pp. 81-+, Oct., 2004.

[32] L.-S. The, D. D. Clayton, R. Diehl et. al., A\&A 450 (May, 2006) 1037-1050 [astro-ph/0601039].

[33] S. E. Woosley, R. E. Taam and T. A. Weaver, ApJ 301 (Feb., 1986) 601-623.

[34] M. D. Leising ApJ 563 (2001) 185-190.

[35] P. Lundqvist, C. Kozma, J. Sollerman and C. Fransson, A\&A 374 (Aug., 2001) 629-637 [arXiv:astro-ph/0105402].

[36] S. M. Matz and G. H. Share, ApJ 362 (Oct., 1990) 235-240.

[37] G. G. Lichti, K. Bennett, J. W. den Herder et. al., A\&A 292 (Dec., 1994) 569-579.

[38] M. D. Leising, W. N. Johnson, J. D. Kurfess et. al., ApJ 450 (Sept., 1995) 805-+.

[39] D. J. Morris, K. Bennett, H. Bloemen et. al., in AIP Conf. Proc. 410: Proceedings of the Fourth Compton Symposium, pp. 1084-+, Nov., 1997.

[40] R. Georgii, S. Plüschke, R. Diehl et. al., A\&A 394 (Nov., 2002) 517-523.

[41] D. D. Clayton and F. Hoyle, ApJ 187 (Feb., 1974) L101+.

[42] M. D. Leising and D. D. Clayton, ApJ 323 (1987) 159-169.

[43] M. D. Leising, G. H. Share, E. L. Chupp and G. Kanbach, ApJ 328 (May, 1988) 755-762. 
[44] A. F. Iyudin, K. Bennett, H. Bloemen et. al., A\&A 300 (Aug., 1995) 422-+.

[45] M. Hernanz, J. José, A. Coc et. al., ApJ 526 (1999) L97-L100.

[46] P. Martin and J. Vink, New Astronomy Review 52 (Oct., 2008) 401-404.

[47] F. A. Harrison, F. E. Christensen, W. Craig et. al., Experimental Astronomy 20 (Dec., 2005) 131-137.

[48] P. Ferrando, M. Arnaud, U. Briel et. al., Memorie della Societa Astronomica Italiana 79 (2008) 19-+.

[49] M. Renaud, F. Lebrun, A. Decourchelle et. al., Memorie della Societa Astronomica Italiana 79 (2008) $50-+[0709.2648]$. 